\title{
Dynamic Modeling of Portfolio Credit Risk with Common Shocks
}

\author{
Areski Cousin \\ ISFA, Université Lyon 1 \\ AFFI Spring 2011 International Meeting
}

Montpellier, 12 May 2011

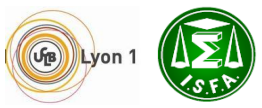




\section{Introduction}

Tom Bielecki, Areski Cousin, Stéphane Crépey and Alexander Herbertsson Dynamic Modeling of Portfolio Credit Risk with Common Shocks 
Main issue: hedging of portfolio credit derivatives

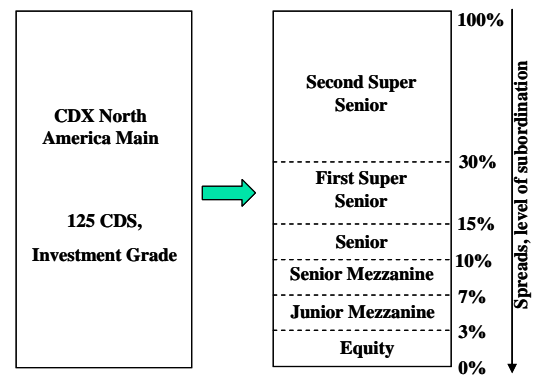

- Cash-flows driven by the realized path of the aggregate loss process

$$
L_{t}=\frac{1}{n} \sum_{i=1}^{n}\left(1-R_{i}\right) H_{t}^{i}
$$

where $R_{i}$ is the recovery rate and $H_{t}^{i}$ is the default indicator of obligor $i$ 


\section{Introduction}

Hedging using the one-factor Gaussian copula model?

Advantages:

- Bottom-up model: account for dispersion of default risk among names in the portfolio

- Copula construction of default times: Calibration of CDS spreads and CDO tranche quotes can be made using two separate numerical procedures

- Factor model: fast algorithms to compute aggregate loss distribution

Drawbacks:

- Static model

- Base correlation approach unable to describe consistently the dependence structure of default times 


\section{Dynamic model of portfolio credit risk}

Simultaneous default model

- Defaults are the consequence of triggering-events affecting simultaneously pre-specified groups of obligors

Example: $n=5$ and $\mathcal{Y}=\{\{1\},\{2\},\{3\},\{4\},\{5\},\{4,5\},\{2,3,4\},\{1,2\}\}$.

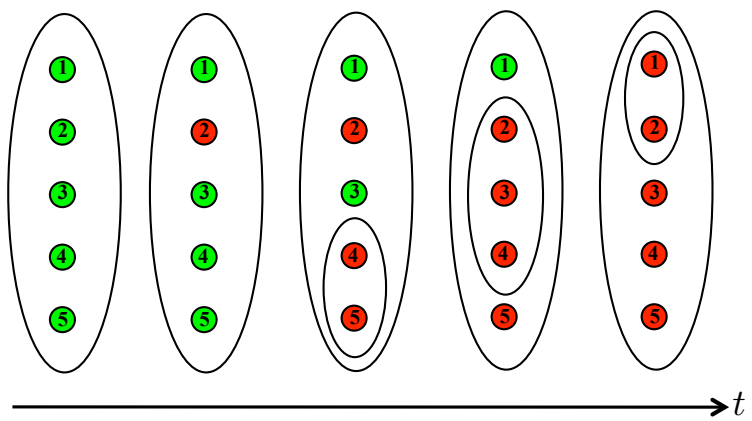




\section{Dynamic model of portfolio credit risk}

- $\{1, \ldots, n\}$ set of credit references

- $\mathcal{Y}=\left\{\{1\}, \ldots,\{n\}, I_{1}, \ldots, I_{m}\right\}$ pre-specified groups of obligors

- $\lambda_{Y}=\lambda_{Y}(t)$ deterministic intensity function of the triggering-event associated with group $Y \in \mathcal{Y}$

- $\mathbf{H}_{t}=\left(H_{t}^{1}, \ldots, H_{t}^{n}\right)$ defined as multivariate continuous-time Markov chain in $\{0,1\}^{n}$ such that for $\mathbf{k}, \mathbf{m} \in\{0,1\}^{n}$ :

$$
\mathbb{P}\left(\mathbf{H}_{t+d t}=\mathbf{m} \mid \mathbf{H}_{t}=\mathbf{k}\right)=\sum_{Y \in \mathcal{Y}} \lambda_{Y}(t) \mathbf{1}_{\left\{\mathbf{k}^{Y}=\mathbf{m}\right\}} d t
$$

where $\mathbf{k}^{Y}$ is obtained from $\mathbf{k}=\left(k_{1}, \ldots, k_{n}\right)$ by replacing the components $k_{j}, j \in Y$, by number one. ex: $(0,1,0,0)^{\{1,2,4\}}=(1,1,0,1)$

- $\mathcal{F}_{t}=\sigma\left(\mathbf{H}_{u}, u \leq t\right)$ natural filtration of $\mathbf{H}$ 


\section{Dynamic model of portfolio credit risk}

Example: $n=2, \mathcal{Y}=\{\{1\},\{2\},\{1,2\}\} . \mathbf{H}_{t}=\left(H_{t}^{1}, H_{t}^{2}\right)$ is a multivariate continuous-time Markov chain with space set $\{(0,0),(1,0),(0,1),(1,1)\}$ and generator matrix

$$
\begin{gathered}
(0,0) \\
(1,0) \\
(0,1)
\end{gathered}\left(\begin{array}{cccc}
- & \lambda_{\{1\}} & \lambda_{\{2\}} & \lambda_{\{1,2\}} \\
0 & - & 0 & \lambda_{\{2\}}+\lambda_{\{1,2\}} \\
0 & 0 & - & \lambda_{\{1\}}+\lambda_{\{1,2\}} \\
0 & 0 & 0 & 0
\end{array}\right)
$$

- Obligor 1 defaults with intensity $\lambda_{\{1\}}+\lambda_{\{1,2\}}$ regardless of the state of the pool

- Obligor 2 defaults with intensity $\lambda_{\{2\}}+\lambda_{\{1,2\}}$ regardless of the state of the pool

- No contagion effect : Past defaults do not have any effect on intensities of surviving names 


\section{Dynamic model of portfolio credit risk}

General case: Obligor $i$ defaults with intensity $\eta_{i}(t)=\sum_{Y \in \mathcal{Y}} \lambda_{Y}(t) \mathbf{1}_{\{i \in Y\}}$

$$
\mathbb{P}\left(H_{t+d t}^{i}-H_{t}^{i}=1 \mid \mathcal{F}_{t}\right)=\mathbb{P}\left(H_{t+d t}^{i}-H_{t}^{i}=1 \mid H_{t}^{i}\right)=\left(1-H_{t}^{i}\right) \eta_{i}(t) d t
$$

- Each default indicator $H^{i}, i=1, \ldots, n$ is a Markov process with respect to $\mathcal{F}$ 
Separate calibration procedure of CDS-s and CDO tranches

For any $i=1, \ldots, n$, the price at time $t$ of a CDS referencing name $i$ (European-type payoff):

$$
\mathbb{E}\left[\Phi\left(H_{T}^{i}\right) \mid \mathcal{F}_{t}\right]=\mathbb{E}\left[\Phi\left(H_{T}^{i}\right) \mid H_{t}^{1}, \ldots, H_{t}^{n}\right]=\mathbb{E}\left[\Phi\left(H_{T}^{i}\right) \mid H_{t}^{i}\right]
$$

Hedging CDO tranches with single-name CDS

- Derive price dynamics of CDO tranche and single-name CDS-s

- Computation of min-variance hedging strategies in this incomplete market model

- But: price of portfolio loss derivatives solves a large system of Kolmogorov backward equations that is numerically intractable at least for large portfolios $(n>20)$ 


\section{Common-Shock Model Interpretation}

- For any pre-specified group $Y \in \mathcal{Y}=\left\{\{1\}, \ldots,\{n\}, I_{1}, \ldots, I_{m}\right\}$, we define

$$
\tau_{Y}=\inf \left\{t \geq 0 \mid \int_{0}^{t} \lambda_{Y}(s) d s>E_{Y}\right\}
$$

where $E_{Y}, Y \in \mathcal{Y}$ are independent and exponentially distributed random variables with parameter 1 .

- $\tau_{Y}$ is the arrival time of shock $Y$ that yields default of non-defaulted names in group $Y$

- Default time of name $i=1, \ldots, n$ defined by:

$$
\widehat{\tau}_{i}=\min _{\{Y \in \mathcal{Y} ; i \in Y\}} \tau_{Y}
$$

\section{Common-Shock Model Interpretation}

For all $t_{1}, \ldots, t_{n} \geq 0$, the following relation holds

$$
\mathbb{P}\left(\widehat{\tau}_{1}>t_{1}, \ldots, \widehat{\tau}_{n}>t_{n}\right)=\mathbb{P}\left(\tau_{1}>t_{1}, \ldots, \tau_{n}>t_{n}\right)
$$

where $\tau_{i}:=\inf \left\{t \geq 0 \mid H_{t}^{i}=1\right\}$ is the default time of name $i$ in the Markovian model 


\section{Common-Shock Model Interpretation}

Using fast recursion procedure for pricing and hedging CDO tranches

- Thanks to the common-shock model interpretation:

$$
L_{t}=\frac{1}{n} \sum_{i=1}^{n}\left(1-R_{i}\right) \mathbf{1}_{\left\{\tau_{i} \leq t\right\}} \stackrel{d}{=} \frac{1}{n} \sum_{i=1}^{n}\left(1-R_{i}\right) \mathbf{1}_{\left\{\widehat{\tau}_{i} \leq t\right\}}
$$

- where $\mathbf{1}_{\left\{\widehat{\tau}_{1} \leq t\right\}}, \ldots, \mathbf{1}_{\left\{\widehat{\tau}_{n} \leq t\right\}}$ are conditionally independent Bernoulli's given common-shock indicators $\mathbf{1}_{\left\{\tau_{I_{1}} \leq t\right\}}, \ldots, \mathbf{1}_{\left\{\tau_{I_{m}} \leq t\right\}}$

- For any state of the Markov process $\mathbf{H}_{t}$, there exists an "equivalent" common-shock model that matches joint distribution of default times for non-defaulted names.

- Computation of min-variance hedging strategies is also tractable 


\section{Common-Shock Model Interpretation}

\section{Two-step calibration procedure}

\section{First step:}

- CDS spread of name $i$ at time $t=0$ can be expressed as a function of survival probabilities $\mathbb{P}\left(\tau_{i}>t\right)=\exp \left(-\int_{0}^{t} \eta_{i}(u) d u\right)$ where

$$
\eta_{i}(u)=\lambda_{\{i\}}(u)+\sum_{k=1}^{m} \lambda_{I_{k}}(u) \mathbf{1}_{\left\{i \in I_{k}\right\}}
$$

- Marginal default intensities $\eta_{i}, i=1, \ldots, n$, can be calibrated on single-name CDS curves using a standard bootstrap procedure

\section{Second step:}

- Common-shock intensities $\lambda_{I_{k}}, k=1, \ldots, m$ are calibrated on CDO tranche quotes using the recursion algorithm 


\section{Calibration on CDX index}

Data set: 5-year CDX North-America IG index on 20 December 2007

- Quoted spreads at different pillars of the $n=125$ index constituents

- Quoted spreads of standard tranches $[0,3],[3,7],[7,10],[10,15],[15,30]$

\section{Model specification:}

- Names are labelled with respect to decreasing level of spreads

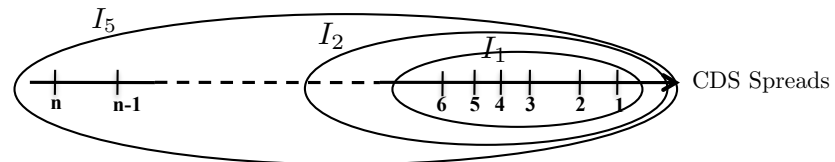

- $m=5$ groups $I_{1} \subset \cdots \subset I_{5}$ such that $I_{1}=\{1, \ldots, 6\}, I_{2}=\{1, \ldots, 19\}$, $I_{3}=\{1, \ldots, 25\}, I_{4}=\{1, \ldots, 61\}, I_{5}=\{1, \ldots, 125\}$

- Piecewise-constant intensities $\lambda_{\{1\}}, \ldots, \lambda_{\{125\}}, \lambda_{I_{1}}, \ldots, \lambda_{I_{5}}$ with grid points corresponding to CDS pillars

- Homogeneous and constant recovery rates: $40 \%$

- Constant short-term interest rate: $3 \%$ 


\section{Calibration on CDX index}

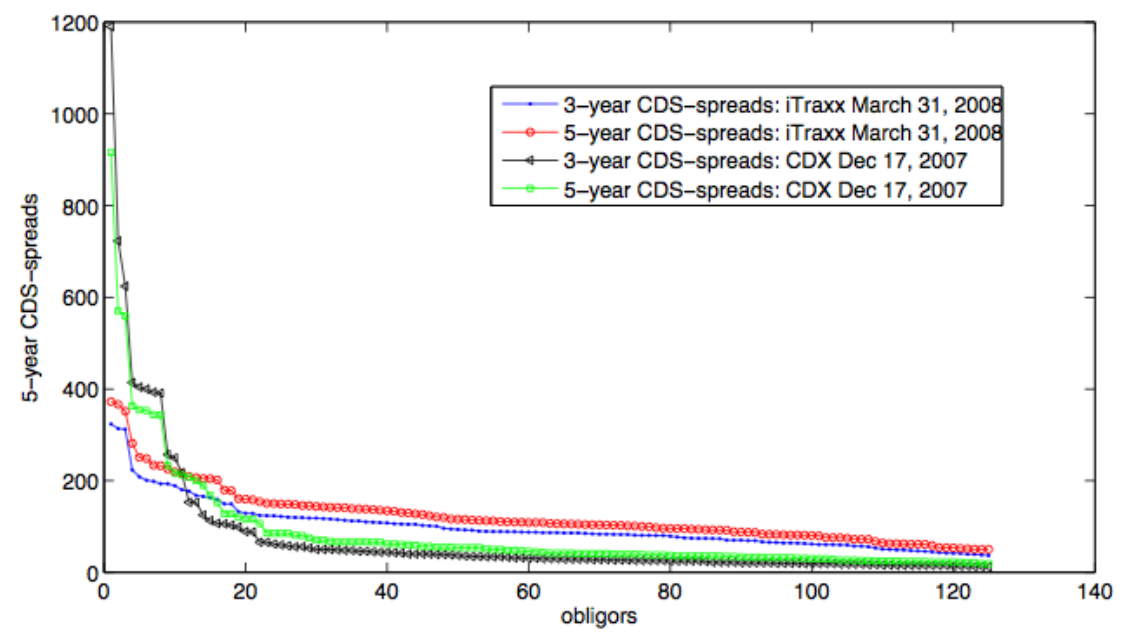




\section{Calibration on CDX index}

Calibration results:

\begin{tabular}{|c||c|c|c|c|c|}
\hline Tranche & {$[0,3]$} & {$[3,7]$} & {$[7,10]$} & {$[10,15]$} & {$[15,30]$} \\
\hline \hline Model spread in bps & 48.0701 & 254.0000 & 124.0000 & 61.0000 & 38.9390 \\
Market spread in bps & 48.0700 & 254.0000 & 124.0000 & 61.0000 & 41.0000 \\
Abs. Err. in bps & 0.0001 & 0.0000 & 0.0000 & 0.0000 & 2.0610 \\
\% Rel. Err. & 0.0001 & 0.0000 & 0.0000 & 0.0000 & 5.0269 \\
\hline
\end{tabular}

- Names in the set $I_{5} \backslash I_{4}$ are excluded from the calibration constraints (they can only default within the Armageddon shock $I_{5}$ ) 


\section{Calibration on CDX index}

\section{Implied 5-year loss distribution:}

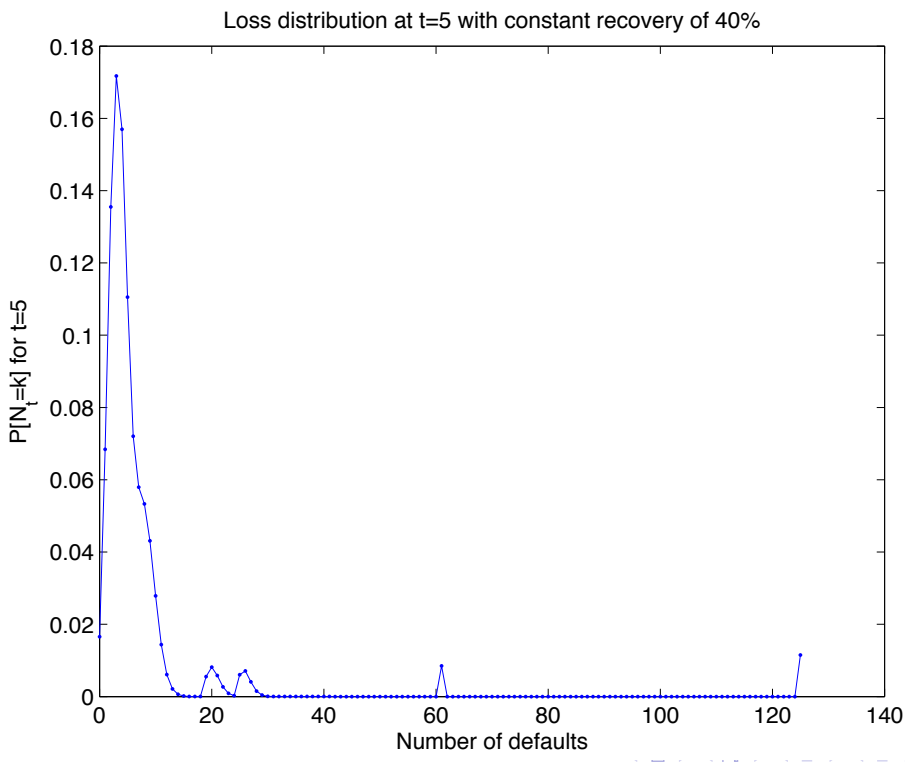




\section{Min-variance hedging strategies}

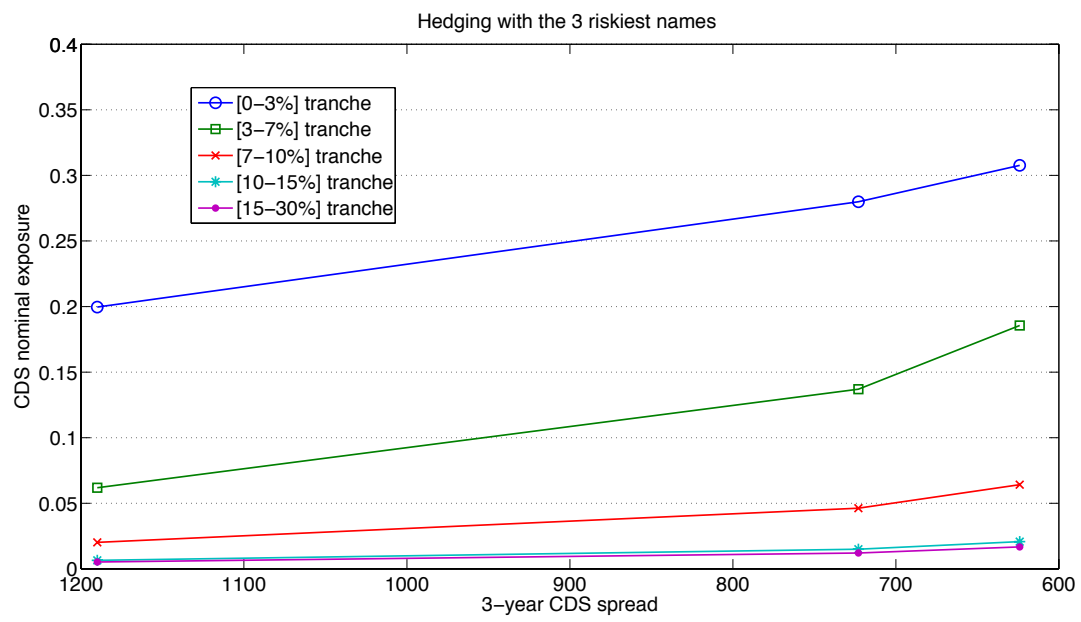




\section{Min-variance hedging strategies}

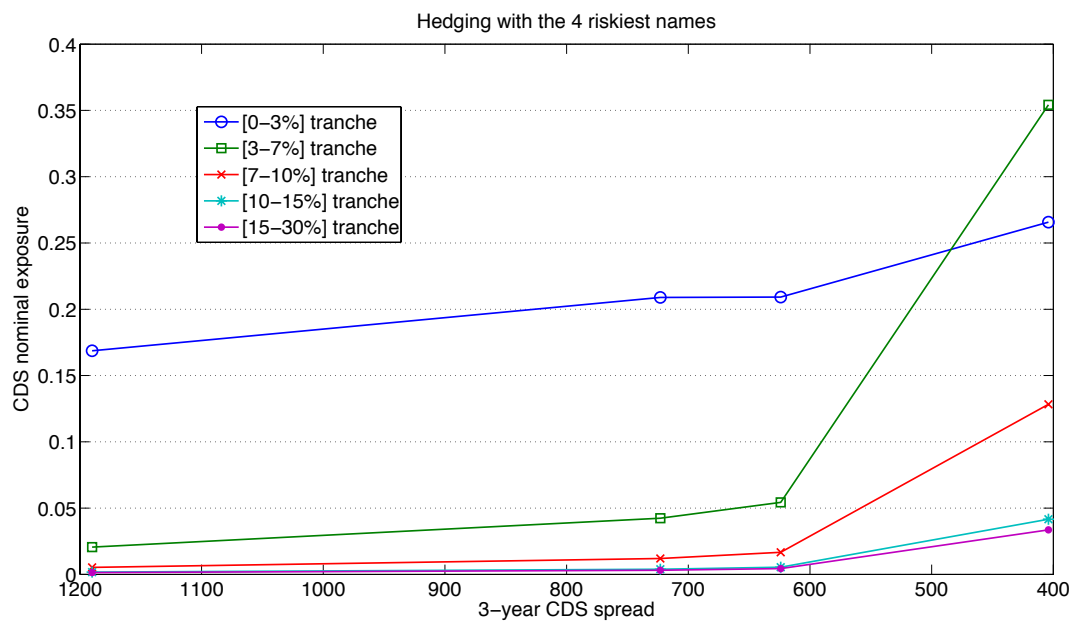




\section{Min-variance hedging strategies}

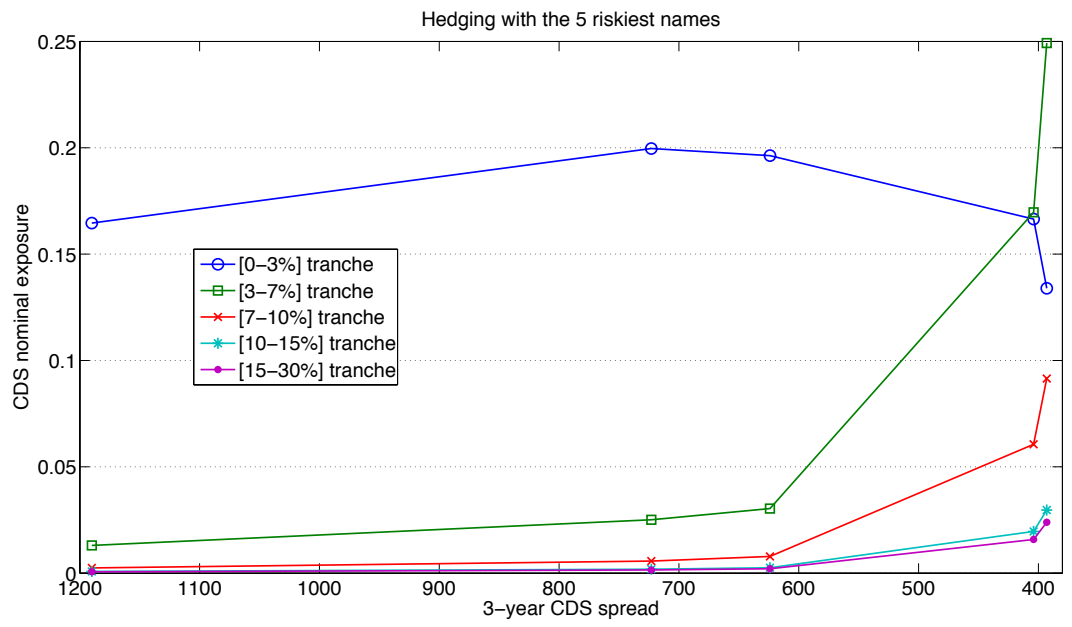




\section{Min-variance hedging strategies}

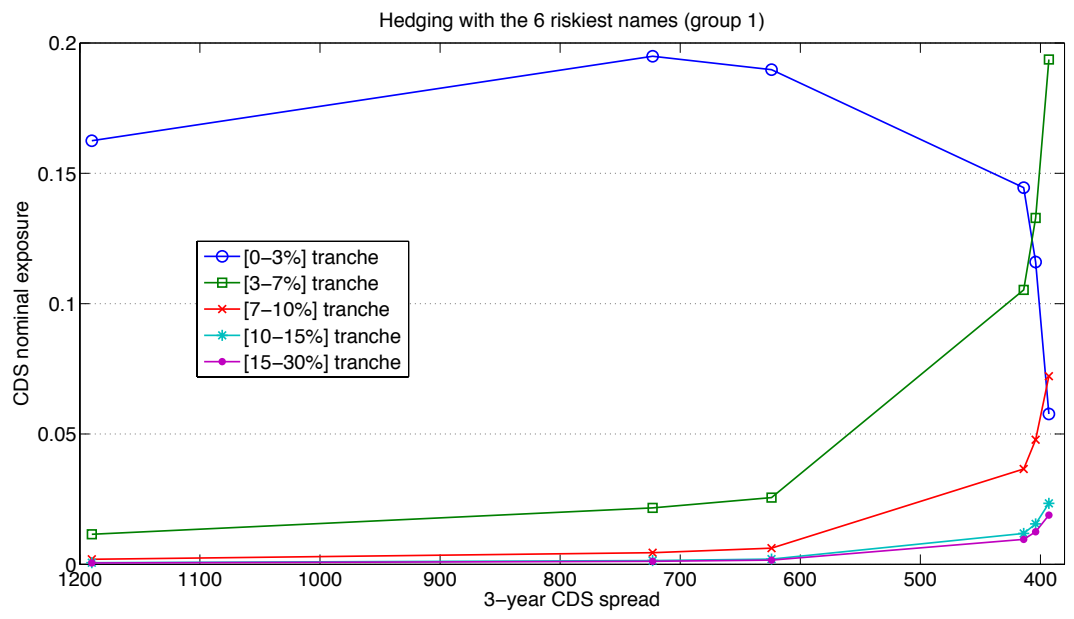




\section{Conclusion}

Thank you for your attention! 


\section{References}

衰 Bielecki, T.R., Vidozzi, A. And Vidozzi, L.: A Markov Copulae Approach to Pricing and Hedging of Credit Index Derivatives and Ratings Triggered Step-Up Bonds, J. of Credit Risk, 2008.

词 Brigo, D., Pallavicini, A., Torrential, R.: Calibration of CDO Tranches with the Dynamical Generalized-Poisson Loss Model. Working Paper, 2006.

诖 Elouerkhaoui, Y.: Pricing and Hedging in a Dynamic Credit Model. International Journal of Theoretical and Applied Finance, Vol. 10, Issue 4, 703-731, 2007.

言 Lindskog, F. And McNeil, A. J.: Common Poisson Shock Models: Applications to Insurance and Credit Risk Modelling. ASTIN Bulletin, 33(2), 209-238, 2003 